\title{
Design of Linear Functional Observer for MIMO LTI Systems
}

\author{
Prakash K. Nakade \\ Assistant Professor, Insru. Dept, KGCE, \\ Karjat Mumbai University.
}

\author{
Girish G. Galgate \\ Assistant Professor, Extc. Dept., KGCE, \\ Karjat, Mumbai University
}

\begin{abstract}
This paper presents an efficient technique to design reduce order linear functional observer for linear time invariant systems. Assuming the existence of linear state feedback controller to achieve stability or some control performance criteria of the linear system, a design procedure is proposed for reconstruction of the state feedback control action. The attractive features of the proposed design procedure are that the resulted linear state functional state observer is of a very low order and it requires information of a small number of outputs. The proposed observer asymptotically converges to any number of linear functions. Numerical examples are considered to illustrate the properties of the observer.
\end{abstract}

\section{INTRODUCTION}

Estimating linear functional of state vector has been the focus of many researchers over the years. A number of procedures have been proposed to design linear functional state observer. Fairman and Gupta (1980), O'Reilly (1983), Aldeen and Trinh (1999) and Trinh and $\mathrm{Ha}$ (2000)). It is often convenient when designing feedback control system to assume initially that the entire state vector of the system to be controlled is available for feedback.

If the entire state vector can not be measured, in most complex systems, the control law cannot be implemented. In such a case either a new approach that directly accounts for the nonavailability of the entire state vector must be devised, or a suitable approximation to the state vector must be determined. In almost every situation the latter approach, that of developing and using an approximate state vector is vastly simpler than a new direct attack on the design problem. The possibility of constructing an observer was first explored by Bass and Gupta [1] and Luenberger [2, 3].Alterative design procedures for observing a scalar linear function of the state of a multiple output system are minimal partial realization theory and decision method respectively. Design procedures are suggested for observing a scalar linear function of a state of a multiple output system in Moore [4] Murdoc [5] and Roman [6]. Fortmann and Williamson [7] are the first to reconstruct vector linear functionals of the state for multiple output systems. Another method based on the reduction of a state observer for a system in form, where the state functional are treated as additional outputs, was proposed by Fairmann and Gupta.[8] Fairmann and Gupta suggest another method based on reduction of state observer for a system in Luenberger companion form, where the state functional are treated as additional outputs. Tsui [9] developed an interesting algorithm for the design of multifunctional observer. The same author [10], showed that the order of observer can be reduced to the sum of descending ordered observability indices of the system minus the number of the required functional. O'Reilly [11], provides an excellent notes on observer theory in chapter 3 . In this paper, a new observer capable of asymptotically estimating any vector state functional, is introduced the order of the observer is dependent on the ratio of the number of independent output measurements to the number of independent outputs. Same method we can extend for time delay and system with unknown inputs.

The order of proposed observer could be as low as proposed by Tsui and Murdoch. The procedure needs no linear transformation and can be easily developed by a simple MATLAB.m function.

The paper is organized as follows:

The problem statement is given in section 2.The design of linear functional observer is given in section 3. Closed loop properties is included in section 4.A numerical example is included in section 5. Finally, a conclusion is given in section 6 .

\section{PROBLEM STATEMENT}

Consider a time-invariant linear system described by

$$
\begin{aligned}
& (\mathrm{t})=\mathrm{Ax}(\mathrm{t})+\mathrm{Bu}(\mathrm{t}) \\
& \mathrm{Y}(\mathrm{t})=\mathrm{C} \mathrm{x}(\mathrm{t})
\end{aligned}
$$

Where vectors $\mathrm{x}(\mathrm{t}) \varepsilon \mathrm{R} \mathrm{n}, \mathrm{u}(\mathrm{t}) \varepsilon \mathrm{R} \mathrm{m}$ and $\mathrm{y}(\mathrm{t}) \varepsilon \mathrm{R} \mathrm{r}$ are the state, input and output vectors respectively. Matrices

$\mathrm{A} \varepsilon \mathrm{R} \mathrm{n} \times \mathrm{n}, \quad \mathrm{B} \varepsilon \mathrm{R} \mathrm{n} \times \mathrm{m}$ and

$\mathrm{C} \varepsilon \mathrm{R} \mathrm{r} \times \mathrm{n}$ are constants. We assume, without loss of generality, matrix $\mathrm{C}$ takes the following canonical form.

$$
\mathrm{C}=\left[\begin{array}{ll}
\mathrm{C} 1 & 0
\end{array}\right],
$$

Where $\mathrm{C} 1 \varepsilon \mathrm{Rr} \times \mathrm{r}$ is of full rank.

Let us assume that linear state feedback control law

$\mathrm{u}(\mathrm{t})=\mathrm{F} \times(\mathrm{t})$, where $\mathrm{F} \varepsilon \mathrm{R} \mathrm{m} \times \mathrm{n}$ 
has been adopted for system (1) to achieve system stability and/or specified performance objective.

\section{LINEAR FUNCTIONAL OBSERVER} DESIGN

Let the feedback control matrix $\mathrm{F}$ be partitioned as

$\mathrm{F}=\mathrm{KT}+\mathrm{WC}$

where $\mathrm{K} \varepsilon \mathrm{R} \mathrm{m} \times \mathrm{p}, \mathrm{T} \varepsilon \mathrm{R} \mathrm{p} \times \mathrm{n}, \mathrm{W} \varepsilon \mathrm{R} \mathrm{m} \times \mathrm{r}$ and $\mathrm{m} \leq \mathrm{p} \leq \mathrm{n}$ by using (3), the feedback control law, equ . (2), becomes

$\mathrm{u}(\mathrm{t})=\mathrm{Fx}(\mathrm{t})=\mathrm{KT} \mathrm{T}(\mathrm{t})+\mathrm{WCX}(\mathrm{t})$

$=\mathrm{KT} x(\mathrm{t})+\mathrm{W} \mathrm{y}(\mathrm{t})$

$$
=\mathrm{Kz}(\mathrm{t})+\mathrm{W} \mathrm{y}(\mathrm{t})
$$

(5)

$$
\text { Where } \mathrm{z}(\mathrm{t})=\mathrm{T} x(\mathrm{t}) \varepsilon \mathrm{R} \mathrm{p}
$$

subject to

$\dot{z}(t)=E z(t)+T B u(t)+G y(t)$

where $\mathrm{E} \varepsilon \mathrm{R} \mathrm{p} \times \mathrm{p}$ and $\mathrm{G} \varepsilon \mathrm{R} \mathrm{p} \times \mathrm{r}$

As mentioned earlier $\mathrm{E}$ can be chosen to be any matrix with a set of stable eigenvalues, a state forward choice of $\mathrm{E}$ is a diagonal matrix

i .e. $E=\operatorname{diag}(\lambda i) ; i=1,2,--, p$.

Letting e $(t)$ be the estimation error

$\mathrm{e}(\mathrm{t})=\mathrm{z}(\mathrm{t})-\mathrm{Tx}(\mathrm{t})$

Taking the derivatives of equ. (7), gives

$$
\begin{aligned}
\dot{\mathrm{e}}(\mathrm{t})= & \dot{z}(\mathrm{t})-\mathrm{T} \square(\mathrm{t}) \\
= & \mathrm{Ez}(\mathrm{t})+\mathrm{TBu}(\mathrm{t})+\mathrm{G} \mathrm{y}(\mathrm{t}) \\
& -\mathrm{TAx}(\mathrm{t})-\mathrm{TBu}(\mathrm{t}) \\
= & \mathrm{Ez}(\mathrm{t})+\mathrm{GCx}(\mathrm{t})-\mathrm{TAx}(\mathrm{t}) \\
= & \mathrm{Ez}(\mathrm{t})-\mathrm{ETx}(\mathrm{t})+\mathrm{GCx}(\mathrm{t})-\mathrm{TAx}(\mathrm{t})+ \\
& \mathrm{ETx}(\mathrm{t})
\end{aligned}
$$

equs. (3) and (8) imply that (6) can act as linear functional observer for $\mathrm{u}(\mathrm{t})=\mathrm{Fx}(\mathrm{t})$ provided that matrix $\mathrm{E}$ is chosen to be stable, matrices $\mathrm{G}$ and $\mathrm{T}$ fulfill the following constraints:

$\mathrm{GC}-\mathrm{TA}+\mathrm{ET}=0$
$\mathrm{F}=\mathrm{KT}+\mathrm{WC}$

$\mathrm{E}$ is stable

Then equ. (8) becomes

$$
\dot{\mathrm{e}}(\mathrm{t})=\mathrm{E} e(\mathrm{t})
$$

Hence the dynamics of $\mathrm{e}(\mathrm{t})$ are governed by matrix E.Therefore, provided that matrix $\mathrm{E}$ is stable and conditions (9) and (10) are satisfied then error function

$\mathrm{e}(\mathrm{t})=[\mathrm{z}(\mathrm{t})-\mathrm{T} \mathrm{x}(\mathrm{t})] \rightarrow 0$

In this paper, we provide a simple procedure for solving (3) and (9) for matrices T, K, W, G and E. We also derive a lower bound on the order of observer, $\mathrm{p}$.

Consider equation (3), let matrices

$\mathrm{F} \varepsilon \mathrm{R} \mathrm{m} \times \mathrm{n}$ and $\mathrm{T} \varepsilon \mathrm{R} \mathrm{p} \times \mathrm{n}$ be expressed as follows:

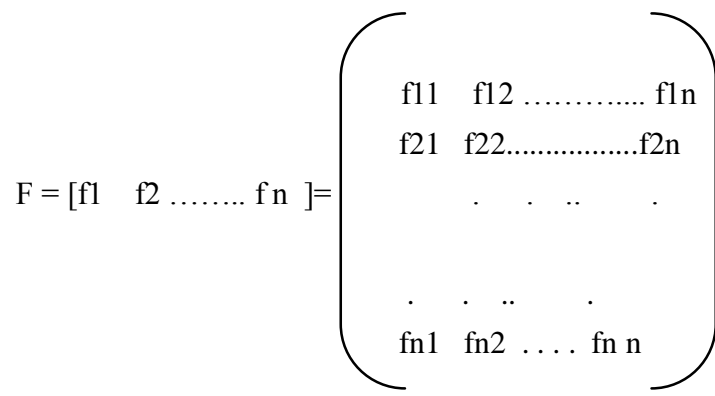

(11)

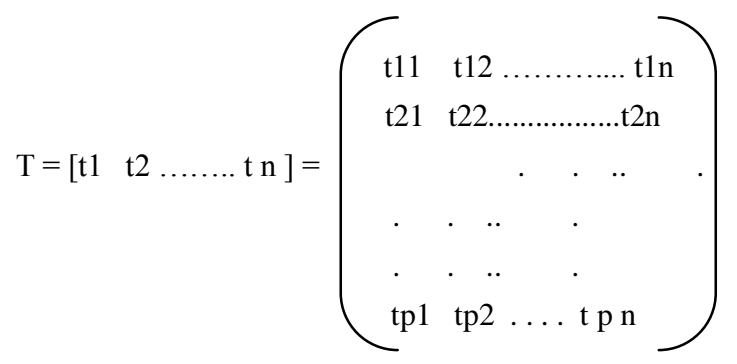

Substituting equations (11) and (12) into equation (3) gives

$\left[\begin{array}{llll}\mathrm{fl} & \mathrm{f} 2 & \ldots & \mathrm{fn}\end{array}\right]-\mathrm{K}\left[\begin{array}{lllll}\mathrm{t} 1 & \mathrm{t} 2 & \ldots . & \mathrm{t} & \mathrm{n}\end{array}\right]-\left[\begin{array}{llll}\mathrm{WC} 1 & 0\end{array}\right]=0$

Using 1(c), (3) and (9) we can write as 
$\mathrm{G}=(\mathrm{TA}-\mathrm{ET}) \quad\left(\begin{array}{c}\mathrm{I} \mathrm{r} \\ 0\end{array}\right] \quad\left(\mathrm{C}_{1}\right)^{-1}$

(TA-ET) $\left(\begin{array}{c}0 \\ \text { In-r }\end{array}\right)=0$

Or

[T1 T2] $\left(\begin{array}{l}\mathrm{A} 12 \\ \mathrm{~A} 22\end{array}\right)-\mathrm{ET} 2=0$

Where A12 $\varepsilon \mathrm{R} \mathrm{r} \times(\mathrm{n}-\mathrm{r}), \mathrm{A} 22 \varepsilon \mathrm{R}(\mathrm{n}-\mathrm{r}) \times(\mathrm{n}-\mathrm{r})$,

$\mathrm{T} 1 \varepsilon \quad \mathrm{R} p \times \mathrm{r}, \mathrm{T} 2 \varepsilon \mathrm{R} \mathrm{p} \times(\mathrm{n}-\mathrm{r})$

Equ. (15b) can be written as

$\Psi \mathrm{t}=0$

Where

$\Psi=\left[\begin{array}{ccccccc}\text { A T12 } & \text { A T } 22 & -\lambda 1 \text { In-r } & 0 & 0 & & 0 \\ 0 & 0 & \text { A T12 } & \text { A T22 } & -\lambda 1 \text { In-r } & 0 \\ \cdot & \cdot & . & \cdot & & \text {. } \\ 0 & 0 & 0 & 0 & & 0\end{array}\right.$

\begin{tabular}{|c|c|}
\hline 0 & 0 \\
\hline 0 & 0 \\
\hline • & • \\
\hline AT1 & $\lambda p$ In-r \\
\hline
\end{tabular}

(15d)

Using equ. (3), we can partitioned as

$\mathrm{WC} 1=(\mathrm{F}-\mathrm{KT})\left(\begin{array}{l}\mathrm{Ir} \\ 0\end{array}\right)$

and

$(\mathrm{F}-\mathrm{KT})\left(\begin{array}{c}0 \\ \text { In-r }\end{array}\right)=0$
From equ (16b), gives

$\mathrm{F} 2=\mathrm{KT} 2$,

Where F2 $\varepsilon \mathrm{R} m \times(\mathrm{n}-\mathrm{r})$

In matrix form equation (16) can be written as

$$
\Omega \mathrm{t}=\mathrm{f}
$$

Where

$$
\begin{aligned}
& \Omega=\operatorname{diag}\{\mathrm{K}\}, \quad \Omega \in \mathrm{R} \mathrm{m}(\mathrm{n}-\mathrm{r}) \times \mathrm{p}(\mathrm{n}-\mathrm{r}) \\
& \mathrm{t}=[\mathrm{t} 1,1 \quad \mathrm{t} 1,2, \mathrm{t} 1, \mathrm{n} \cdot \mathrm{t} 2,1 . \mathrm{t} 2, \mathrm{n} \cdot \mathrm{tp}, \mathrm{n}-1 . \mathrm{t} \mathrm{p}, \mathrm{n}] \mathrm{T} \varepsilon \mathrm{R} \mathrm{p} \mathrm{n} \\
& \quad \text { and }
\end{aligned}
$$

$\mathrm{f}=[\mathrm{f} 1, \mathrm{r}+1 \mathrm{fl}, \mathrm{r}+2 \ldots \mathrm{f} 1 \mathrm{n} \mathrm{f} 2, \mathrm{r}+1 \ldots \mathrm{f} 2, \mathrm{r}+\mathrm{n}$ fm,n-1 ..fm, n]T $\varepsilon \mathrm{R} \mathrm{m}$ n

Eqn.17 (a) represents m (n-r) eqns. with p (n-r) unknowns.

As $p \geq m,(p-m)$ elements of $t i, i=r+1, r+2, \ldots, n$ can be chosen arbitrarily. From (15c) and (17a) we get,

$$
\Pi \mathrm{t}=\mathrm{f}
$$

Where

$\Pi=\left(\begin{array}{l}\Psi \\ \Omega\end{array}\right)$

Equn contains a set of $\{m(n-r)+p(n-r)\}$ with pn unknowns. Thus, a solution exist if and only $\mathrm{p} n \geq m(n-r)+p(n-r)$ which implies

$\mathrm{p} \geq \mathrm{m}(\mathrm{n}-\mathrm{r}) / \mathrm{r}$

Where $\mathrm{p}$ is order of observer.

Once $\mathrm{T}$ is found, matrix $\mathrm{W}$ can be determined from equ.( $3 \mathrm{~b})$ and matrix $G$ can be determined from equ.(14),.As a result, all of the observer(6) and control law(2) parameters are determined and construction of observer is complete.

Design algorithm: (Construction of a functional observer for $\mathrm{u}(\mathrm{t})$ $=\mathrm{F} x(\mathrm{t}))$ 
1. Obtain a state feedback controller LQR with control weighting matrices $\mathrm{Q}=2 \mathrm{I} 15$ and $\mathrm{R}=\mathrm{I} 2$.

2. Use condition (19) to obtain order, $p$, of the observer (6).

3. Choose arbitrarily matrix $\mathrm{K}$ as $(\mathrm{m} \times \mathrm{p})$ elements of matrix $\mathrm{F}$.

4. Choose arbitrarily stable matrix E.

5. Obtain matrix $\mathrm{T}$ from equ (18a).

6. Obtain matrix $\mathrm{W}$ from equ. (16b).

7. Obtain matrix $\mathrm{G}$ from equ. (14).

8. By using steps 1-7 above, a linear functional reduced order observer (6) and feedback controller (2) are obtained.

\section{CLOSE LOOP PROPERTIES}

From equations (1b) (2),(4) and(7), give

$\mathrm{u}(\mathrm{t})=\mathrm{Ke}(\mathrm{t})+\mathrm{KT} \mathrm{x}(\mathrm{t})+\mathrm{WC}(\mathrm{x} \mathrm{t})$

\section{CLOSE LOOP PROPERTIES}

Hence close loop system is obtained

$\dot{X}(t)=(A+B F) x(t)+B K e(t)$ and $\dot{e}(t)=E$ e $(t)$

$\left(\begin{array}{c}\dot{x}(t) \\ \dot{e}(t)\end{array}\right)=\left(\begin{array}{cc}A+B F & B K \\ 0 & E\end{array}\right)\left(\begin{array}{l}x(t) \\ e(t)\end{array}\right)$

The eigen values of the observer based closed loop system are the union of the eigen values of the closed loop system without the observer and the eigen values of the observer.

$\mathrm{A}=$

Columns 1 through 10

$\begin{array}{cccccccccc}3.3000 & 0 & -0.6000 & -1.5000 & -0.3000 & 2.2000 & 0 & -0.4000 & -1.0000 & -0.2000 \\ -0.3000 & 6.0000 & 0 & -0.6000 & 1.5000 & -0.2000 & 4.0000 & 0 & -0.4000 & 1.0000 \\ -1.2000 & 1.5000 & 9.0000 & -0.3000 & -3.0000 & -0.8000 & 1.0000 & 6.0000 & -0.2000 & -2.0000 \\ -2.2500 & -0.6000 & -2.4000 & 3.0000 & 0 & -1.5000 & -0.4000 & -1.6000 & 2.0000 & 0 \\ -0.6000 & 1.5000 & -1.5000 & -1.5000 & 3.7500 & -0.4000 & 1.0000 & -1.0000 & -1.0000 & 2.5000 \\ -1.1000 & 0 & 0.2000 & 0.5000 & 0.1000 & -2.2000 & 0 & 0.4000 & 1.0000 & 0.2000 \\ 0.1000 & -2.0000 & 0 & 0.2000 & -0.5000 & 0.2000 & -4.0000 & 0 & 0.4000 & -1.0000 \\ 0.4000 & -0.5000 & -3.0000 & 0.1000 & 1.0000 & 0.8000 & -1.0000 & -6.0000 & 0.2000 & 2.0000 \\ 0.7500 & 0.2000 & 0.8000 & -1.0000 & 0 & 1.5000 & 0.4000 & 1.6000 & -2.0000 & 0 \\ 0.2000 & -0.5000 & 0.5000 & 0.5000 & -1.2500 & 0.4000 & -1.0000 & 1.0000 & 1.0000 & -2.5000 \\ 1.4300 & 0 & -0.2600 & -0.6500 & -0.1300 & -1.1000 & 0 & 0.2000 & 0.5000 & 0.1000 \\ -0.1300 & 2.6000 & 0 & -0.2600 & 0.6500 & 0.1000 & -2.0000 & 0 & 0.2000 & -0.5000 \\ -0.5200 & 0.6500 & 3.9000 & -0.1300 & -1.3000 & 0.4000 & -0.5000 & -3.0000 & 0.1000 & 1.0000 \\ -0.9750 & -0.2600 & -1.0400 & 1.3000 & 0 & 0.7500 & 0.2000 & 0.8000 & -1.0000 & 0 \\ -0.2600 & 0.6500 & -0.6500 & -0.6500 & 1.6250 & 0.2000 & -0.5000 & 0.5000 & 0.5000 & -1.2500\end{array}$


Columns 11 through 15

$\begin{array}{ccccc}-3.3000 & 0 & 0.6000 & 1.5000 & 0.3000 \\ 0.3000 & -6.0000 & 0 & 0.6000 & -1.5000 \\ 1.2000 & -1.5000 & -9.0000 & 0.3000 & 3.0000 \\ 2.2500 & 0.6000 & 2.4000 & -3.0000 & 0 \\ 0.6000 & -1.5000 & 1.5000 & 1.5000 & -3.7500 \\ 1.6500 & 0 & -0.3000 & -0.7500 & -0.1500 \\ -0.1500 & 3.0000 & 0 & -0.3000 & 0.7500 \\ -0.6000 & 0.7500 & 4.5000 & -0.1500 & -1.5000 \\ -1.1250 & -0.3000 & -1.2000 & 1.5000 & 0 \\ -0.3000 & 0.7500 & -0.7500 & -0.7500 & 1.8750 \\ -1.8700 & 0 & 0.3400 & 0.8500 & 0.1700 \\ 0.1700 & -3.4000 & 0 & 0.3400 & -0.8500 \\ 0.6800 & -0.8500 & -5.1000 & 0.1700 & 1.7000 \\ 1.2750 & 0.3400 & 1.3600 & -1.7000 & 0 \\ 0.3400 & -0.8500 & 0.8500 & 0.8500 & -2.1250\end{array}$

$\mathrm{BT}=$

Columns 1 through 10

$\begin{array}{cccccccccc}1.0000 & 0 & 0.5000 & 1.0000 & 0 & 0 & 0 & 0 & 0 & 0 \\ 0 & 0 & 0 & 0 & 0 & -1.0000 & 0 & -0.5000 & -1.0000 & 0\end{array}$

Columns 11 through 15

$\begin{array}{ccccc}-1.0000 & 2.0000 & 0.3000 & 0 & 0 \\ 0 & 0 & 0 & 0 & 1.0000\end{array}$

And $\mathrm{C}=\left[\begin{array}{lll}\mathrm{I} 5 & : & 0\end{array}\right]$

Open loop Eigen values of system are:
$\lambda(\mathrm{A})=$
4.7594
$-3.4990+4.2421 i$
$-3.4990-4.2421 \mathrm{i}$
3.4326 


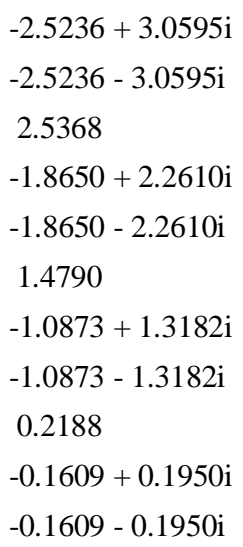

Close loop eigen values are:

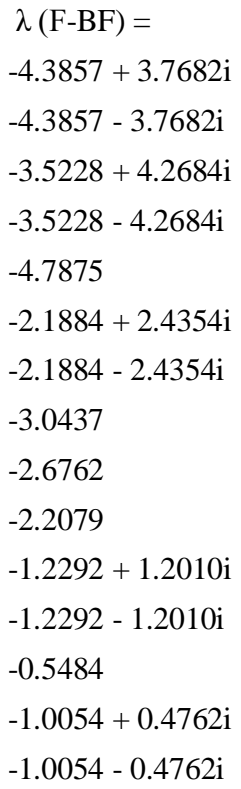

Step 1: System gain matrix is F:

$\mathrm{F}=$

$1.0 \mathrm{e}+003 *$

Columns 1 through 10

$\begin{array}{llllllllll}-0.2858 & 0.0926 & 1.0888 & 0.1921 & -0.5605 & -0.2116 & 0.0688 & 0.8091 & 0.1433 & -0.4160\end{array}$

$\begin{array}{llllllllll}-0.1389 & 0.0209 & 0.6794 & 0.0224 & -0.2577 & -0.1041 & 0.0158 & 0.5044 & 0.0159 & -0.1918\end{array}$

Columns 11 through 15 


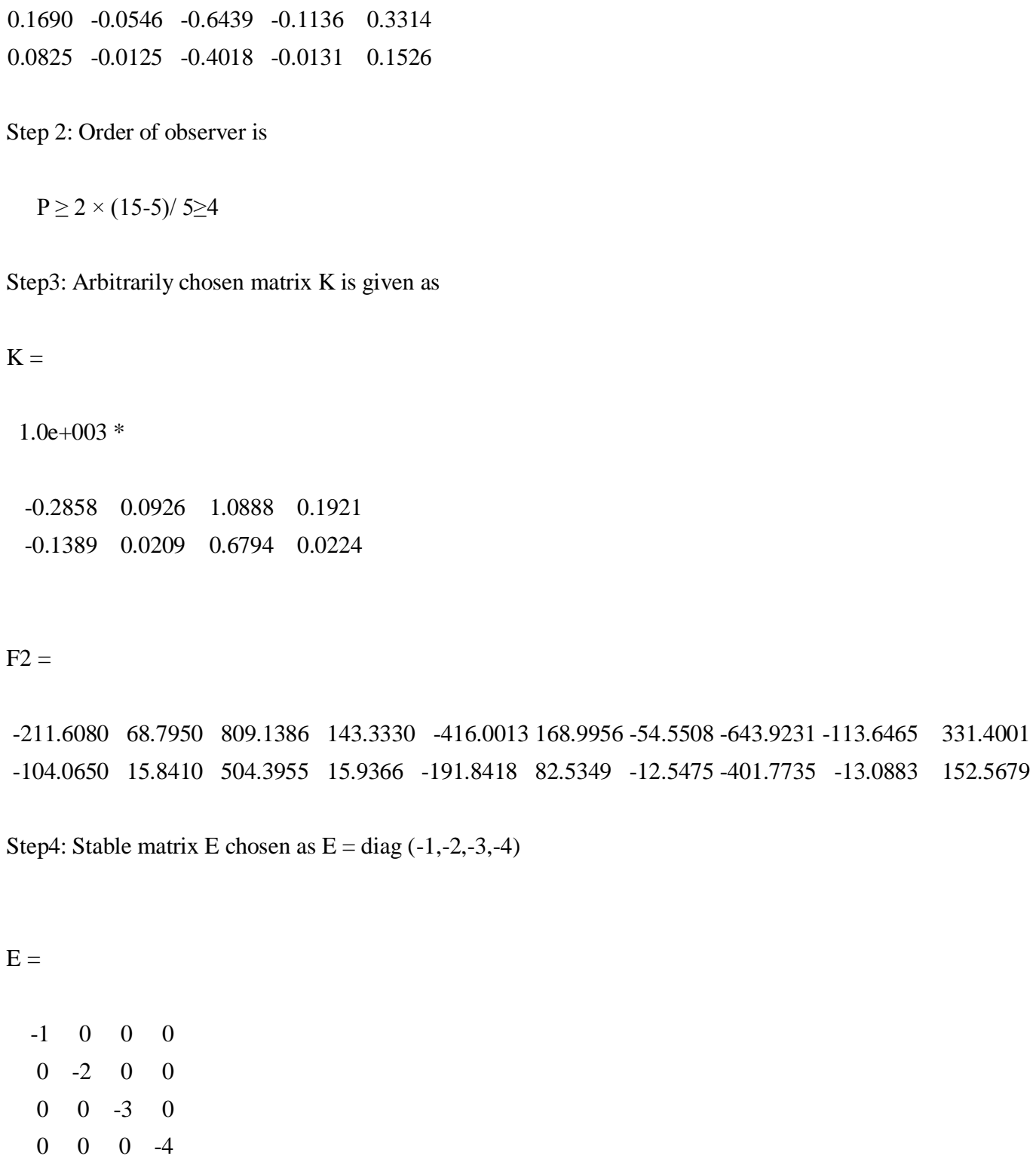

Step5: Obtain matrix T, W, G Matrices

$\mathrm{T}=$

Columns 1 through 10

$\begin{array}{llllllllll}1.0152 & -0.7090 & -6.7476 & -0.2667 & 2.8481 & 1.6454 & -0.9134 & -10.0967 & -0.2127 & 4.1524 \\ 0.0564 & -0.8336 & -8.8909 & 0.3604 & 2.4739 & 0.4073 & -1.5847 & -14.7313 & 2.0720 & 5.0118 \\ 0.0637 & -0.0529 & -0.4838 & -0.0651 & 0.1862 & 0.1632 & -0.1315 & -0.9179 & -0.0800 & 0.4425 \\ 0.0742 & -0.4789 & 0.9375 & -0.0049 & 0.9097 & 0.2250 & 0.5084 & 1.4939 & -0.1158 & -0.9118\end{array}$


Columns 11 through 15

$\begin{array}{ccccc}-0.5115 & 0.5417 & 3.6070 & 0.1990 & -1.7527 \\ 0.4302 & 2.1599 & 3.3620 & 1.1823 & -3.8844 \\ 0.0080 & 0.0529 & 0.0364 & 0.0167 & -0.0576 \\ -0.1343 & -0.8195 & 0.1874 & -0.9597 & 1.3167\end{array}$

$\mathrm{W}=$

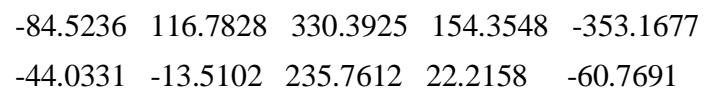

\section{Closed loop responces y $1(\mathrm{t})$ to $y 5(\mathrm{t})$}
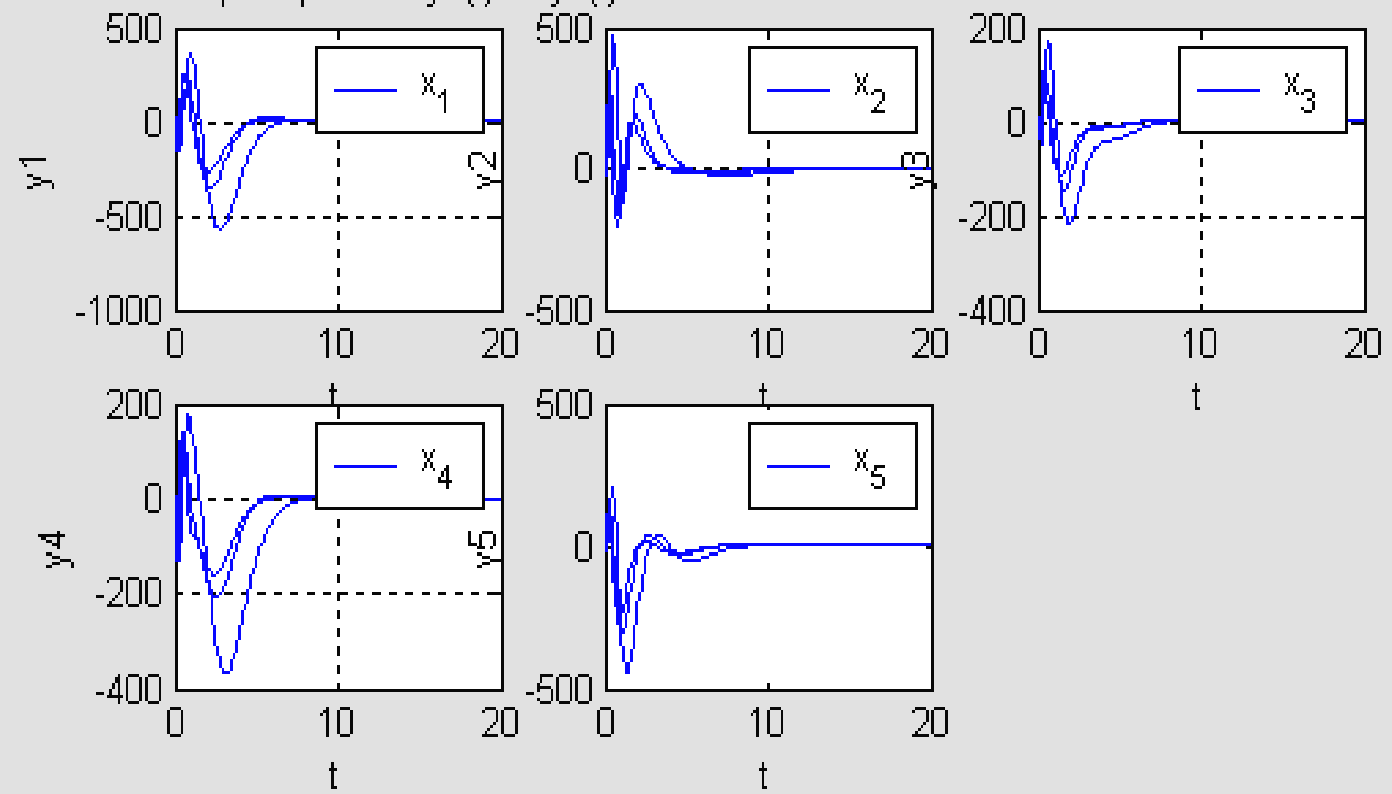

t 


\section{Simulation results:}

When observer is implemented, the following close loop systems results

$$
\left(\begin{array}{c}
\dot{x}(t) \\
\dot{e}(t)
\end{array}\right)=\left(\begin{array}{cc}
A+B W C & B K \\
T B W C+G C & E+T B K
\end{array}\right)\left(\begin{array}{l}
x(t) \\
e(t)
\end{array}\right)
$$

The Eigen values of the observer based closed loop system are the union of the eigen values of the closed loop system without the observer and the eigen values of the observer. To stimulate the dynamic performance of this closed loop system a nonzero initial condition is required. Let us choose, arbitrarily, the following initial condition as

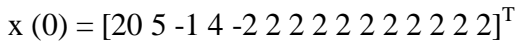

\section{CONCLUSION}

An observer for the estimation of multi functionals of the state vector for linear multivariable control systems is presented in this paper. In addition the observer can serve as estimator for any small subset of the state vector $x$. Numerical examples has been introduced to illustrate the main properties of the proposed observer. In example comparison is provided three control schemes. The schemes are i) direct full state feedback ii) full order observer based controller iii) control scheme proposed in paper. Simulation result shows very little difference among the three schemes.

\section{REFERENCES}

[1] Bass, R. W., and Gura: 'High-order system design via Automatic ControlConference, Atlanta, Georgia, 1965

[2] LUENBERGER, D.G.: 'An introduction to observer', IEEE Tran Auto. Control, 1971 AC-16,pp. 596-602
[3] LUENBERGER, D.G.:'Observers for multi-variable systems', IEEETranAuto.Control, 1966 AC-11, pp.190-197

[4] M00RE, J.B., and LEDWITCH, G.F.:'Minimal order observers for estimating linear functionals of state vector', IEEE Tran Auto. Control, 975 AC-20,p p. 623-632.

[5] MURDOCH,P.:'Observer design of a linear functional of the state vector', IEEE Tran Auto.Control,1973 AC-18,pp.308-310

[6] ROMAN, J.R., JONES, L.E., andBULLOCK,

T.E,: ' Observing a function of the state', Proceedings of IEEE Decision and Control Conference, San Diego, California, 1973.

[7] FORTMAN, T.E., and WILLIAMSON, D.: 'Design of low order observer for linear feedback control laws', IEEE Tran Auto.Control, 1972 AC-18, pp.301-308.

[8] FAIRMAN, F.W., and GUPTA, R.D.,'DDesign of multifunctional reduced order observer'.Int.J.Sys.Sci.,1980,11,pp.1083-1094.

[9] TSUI, C.C.,:'A new algorithm for the design of multifunctional observers', IEEE Tran Auto.Control,1985 AC-30,pp.89-93.

[10] TSUI, C.C.,'on the order reduction of linear functional observers', IEEE TranAuto.Control, 1986 AC-31, pp.447-449.

[11] O'REILLY, J.:'Observe for linear systems'. (Academic Press, 1983)

[12] ALDEEN.'Reduced order linear functional observer for linear system'.IEEE Proc.Control Theory Appl., Vol.146, No.5, 1999.

[13] H.TRINH and Q.HA'Design of linear functional observers for linear systems with unknown inputs'. International Journal of System Sciene.'Vol 31, pp.741-749. 2000.

[14] Q.P. HA. H.TRINH, and G.Dissanayake'A low order linear functional observer for time delayed systems.' Asian control conference 2004 